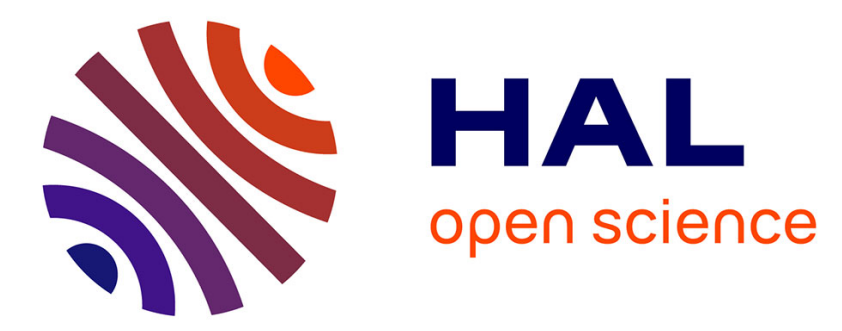

\title{
Coherent combining of high brightness tapered lasers in Master Oscillator Power Amplifier configuration
}

Philipp Albrodt, Marc Hanna, Frédéric Moron, Jonathan Decker, Winterfeldt M., G Blume, Götz Erbert, Paul Crump, Patrick Georges, Gaëlle Lucas-Leclin

\section{- To cite this version:}

Philipp Albrodt, Marc Hanna, Frédéric Moron, Jonathan Decker, Winterfeldt M., et al.. Coherent combining of high brightness tapered lasers in Master Oscillator Power Amplifier configuration. SPIE LASE 2018, Jan 2018, San Francisco, CA, United States. pp.105140T, 10.1117/12.2288337 . hal01782283

\section{HAL Id: hal-01782283 \\ https://hal-iogs.archives-ouvertes.fr/hal-01782283}

Submitted on 1 May 2018

HAL is a multi-disciplinary open access archive for the deposit and dissemination of scientific research documents, whether they are published or not. The documents may come from teaching and research institutions in France or abroad, or from public or private research centers.
L'archive ouverte pluridisciplinaire HAL, est destinée au dépôt et à la diffusion de documents scientifiques de niveau recherche, publiés ou non, émanant des établissements d'enseignement et de recherche français ou étrangers, des laboratoires publics ou privés. 


\title{
Coherent combining of high brightness tapered lasers in Master Oscillator Power Amplifier configuration
}

\author{
P. Albrodt a , M. Hanna a , F. Moron ${ }^{\mathrm{a}}$, \\ J. Decker ${ }^{\mathrm{b}}$, M. Winterfeldt ${ }^{\mathrm{b}}, \mathrm{G}_{\text {. Blume }}{ }^{\mathrm{b}}, \mathrm{G}_{\text {. Erbert }}{ }^{\mathrm{b}}$, P. Crump ${ }^{\mathrm{b}}$, \\ P. Georges ${ }^{a}$ and G. Lucas-Leclin ${ }^{\text {a* }}$
${ }^{a}$ Laboratoire Charles Fabry, Institut d'Optique Graduate School, CNRS, Université Paris-Saclay, Palaiseau, France;
${ }^{\mathrm{b}}$ Ferdinand-Braun-Institut, Leibniz-Institut für Höchstfrequenztechnik (FBH), Berlin, Germany;

*gaelle.lucas-leclin@institutoptique.fr,

\begin{abstract}
Improved diode laser beam combining techniques are in strong demand for applications in material processing. Coherent beam combining (CBC) is the only combining approach that has the potential to maintain or even improve all laser properties, and thus has high potential for future systems. As part of our ongoing studies into CBC of diode lasers, we present recent progress in the coherent superposition of high-power single-pass tapered laser amplifiers. The amplifiers are seeded by a DFB laser at $\lambda=976 \mathrm{~nm}$, where the seed is injected into a laterally single-mode ridge-waveguide input section. The phase pistons on each beam are actively controlled by varying the current in the ridge section of each amplifier, using a sequential hill-climbing algorithm, resulting in a combined beam with power fluctuations of below $1 \%$. The currents into the tapered sections of the amplifiers are separately controlled, and remain constant. In contrast to our previous studies, we favour a limited number of individual high-power amplifiers, in order to preserve a high extracted power per emitter in a simple, low-loss coupling arrangement. Specifically, a multi-arm interferometer architecture with only three devices is used, constructed using $6 \mathrm{~mm}$-long tapered amplifiers, mounted junction up on C-mounts, to allow separate contact to single mode and amplifier sections. A maximum coherently combined power of $12.9 \mathrm{~W}$ is demonstrated in a nearly diffraction-limited beam, corresponding to a $65 \%$ combining efficiency, with power mainly limited by the intrinsic beam quality of the amplifiers. Further increased combined power is currently sought.
\end{abstract}

Keywords: coherent beam combining, tapered laser amplifiers, high-brightness diode lasers

\section{INTRODUCTION}

High power diode lasers provide the optical energy for many laser systems and benefit from a high electrical-to-optical efficiency. Even if the brightness of such systems has been dramatically increased within the last decade, it is still an important challenge to scale the power in a beam close to the diffraction limit. Power scaling of industrial diode laser systems is usually based on incoherent geometrical beam combining technologies and spectral beam combining ${ }^{1,2}$. Geometrical beam combining automatically deviates from the diffraction limit, while power scaling via spectral beam combining is limited by the number of achievable wavelength channels and minimum wavelength spacing. In contrast, coherent beam combining (CBC) consists of the superposition of individual laser beams by constructive interference into one high-power laser beam and maintains a single laser line and a diffraction-limited beam profile ${ }^{3}$. The power and the spatial brightness can be scaled far above from what could be achieved from a single device. While CBC in the $\mathrm{kW}$-range has been successfully demonstrated with different approaches and different laser materials ${ }^{4,5}$, CBC of diode lasers is still limited to much lower power levels. Recent studies of arrays of laterally single mode devices enable around $1 \mathrm{~W}$ per channel, delivering up to $40 \mathrm{~W}$ in a single diffraction limited beam from 47 phase-locked elements ${ }^{6}$. On the one hand, arrays of amplifiers offer an elegant solution to scale the number of active devices, and the phase relation between these elements is usually more stable than for individually mounted emitters as they are exposed to approximately the same fluctuations of the temperature. On the other hand, the achievable power per amplifier of such systems is significantly lower compared to the performance of high power individual emitters. 
We are therefore currently working on Master Oscillator Power Amplifier (MOPA) architectures for CBC based on a small number of individual emitters allowing us to reach comparable power levels with a significantly reduced number of elements and a smaller footprint ${ }^{7}$. We investigate $\mathrm{CBC}$ of tapered laser amplifiers because their high power and excellent beam quality is a perfect starting point for power scaling ${ }^{8}$.

\section{AMPLIFIER CHARACTERIZATION}

\subsection{Standard characterization}

The tapered amplifiers (TPA) were grown on GaAs substrates using MOVPE, fabricated into individual, $6 \mathrm{~mm}$ long devices using i-line lithography. In the studies presented here, we made use of TPAs identical to lasers previously described by Fiebig et al. ${ }^{9}$, but with the internal grating section being replaced by a long $(2 \mathrm{~mm})$ ridge waveguide (RW) section. The tapered section was $4 \mathrm{~mm}$ long $\left(\alpha_{\mathrm{T}}=6^{\circ}\right.$ taper angle). Both facets were passivated and anti-reflection coated. The amplifiers are mounted p-side up on $\mathrm{CuW}$ heat spreaders and $\mathrm{C}$-mounts, with separate electrical contacts used in order to control the injection current of ridge $\left(\mathrm{I}_{\mathrm{rw}}\right)$ and tapered section $\left(\mathrm{I}_{\mathrm{tp}}\right)$ independently. We use C-mounts in order to be able to easily access both facets of the amplifier. The $\mathrm{C}$-mounts were fixed on to a $\mathrm{Cu}$-block that served both as a heatsink and as an optical bench for the collimation optics. The heatsink temperature $\left(\mathrm{T}=20^{\circ} \mathrm{C}\right)$ was actively controlled by a Peltier device mounted on a water cooled radiator. The master oscillator (MO) was a narrow bandwidth DFB laser $(\lambda=976 \mathrm{~nm})$. We present in the following standard test and phase noise characterization of the used amplifiers under representative bias conditions.

The output power characteristic $\mathrm{P}_{\text {out }}\left(\mathrm{I}_{\mathrm{tp}}\right)$ is shown in Figure 1 for a constant ridge current $\mathrm{I}_{\mathrm{rw}}=400 \mathrm{~mA}$. Each of the amplifier elements reaches about $6.5 \mathrm{~W}$ at $10 \mathrm{~A}$ and a heat sink temperature of $20^{\circ} \mathrm{C}$. The output power $\mathrm{P}_{\text {out }}\left(\mathrm{I}_{\mathrm{tp}}\right)$ has a close to linear current dependence when operated in the range $\mathrm{I}_{\mathrm{tp}}=2-9 \mathrm{~A}$. We limit the maximum drive current in the tapered section to $10 \mathrm{~A}$ since it is challenging to efficiently cool the C-Mounts used. The power content in the central lobe (diffraction limited content ${ }^{8,9}$ ) decreases with the current in the tapered section from about $90 \%$ at $3 \mathrm{~A}$ to about $70 \%$ at 10 A reaching about $4.5 \mathrm{~W}$ per device. The electrical to optical efficiency (total power) is higher than $34 \%$ at $7 \mathrm{~A}$ neglecting the power consumed in the ridge section. The output power characteristic $\mathrm{P}_{\text {out }}\left(\mathrm{I}_{\mathrm{rw}}\right)$ shown on the right side in Figure 1 for $\mathrm{I}_{\mathrm{tp}}=7 \mathrm{~A}$ indicates that the power amplifier is mostly saturated in the range of $250-400 \mathrm{~mA}$. We use the ridge current of the amplifiers to correct for the relative phase fluctuations in the multi-arm interferometer to achieve CBC in our experimental setup which is presented later in this proceeding. The relation between the phase piston and the ridge current is close to linear and a current variation of $\Delta \mathrm{I}_{\mathrm{rw}}=35 \mathrm{~mA}$ corresponds to a phase piston of $\Delta \Phi \approx \pi$ in the used TPA ${ }^{10}$. We can comfortably use variations of the ridge current in the range of 250 to $400 \mathrm{~mA}$ with limited impact on the output power per element as shown earlier in Figure 1. Furthermore, we observed a saturation of the output power for a seed power level of $10 \mathrm{~mW}$ meaning that the amplifiers are quite insensitive to slight imbalances of the input power if $\mathrm{P}_{\text {in }}>10 \mathrm{~mW}$.
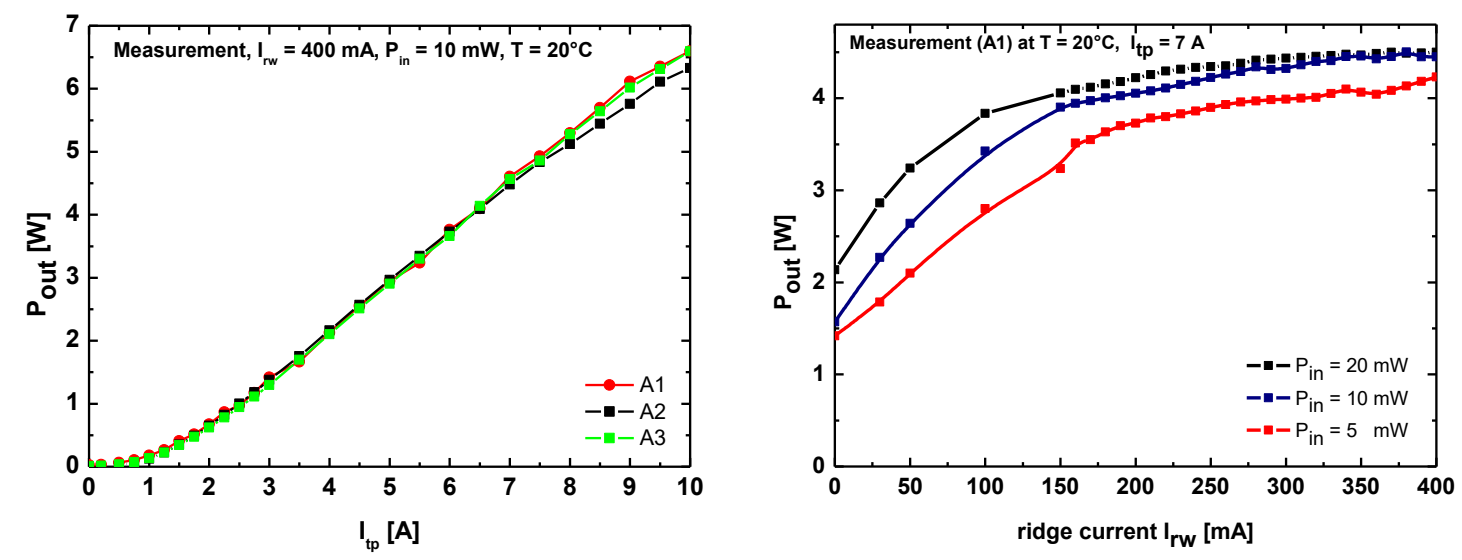

Figure 1: Output power characteristic $\mathrm{P}_{\text {out }}\left(\mathrm{I}_{\mathrm{tp}}\right)$ for three TPAs at constant ridge current (left), output power characteristics $\mathrm{P}_{\mathrm{out}}\left(\mathrm{I}_{\mathrm{rw}}\right)$ of one TPA at constant current in tapered section for different injection powers (right). 


\subsection{Phase noise characterization}

Efficient MOPA-CBC requires a precise control of the relative phases. A mismatch of the phase pistons leads to a decreased combining efficiency and a phase mismatch of $\pi / 30$ leads to loss of about $1 \%$ for CBC of two beams on a beam splitter ${ }^{11}$. It is therefore important to investigate the phase noise in the used TPAs in order to be able to determine the requirements for an active phase control

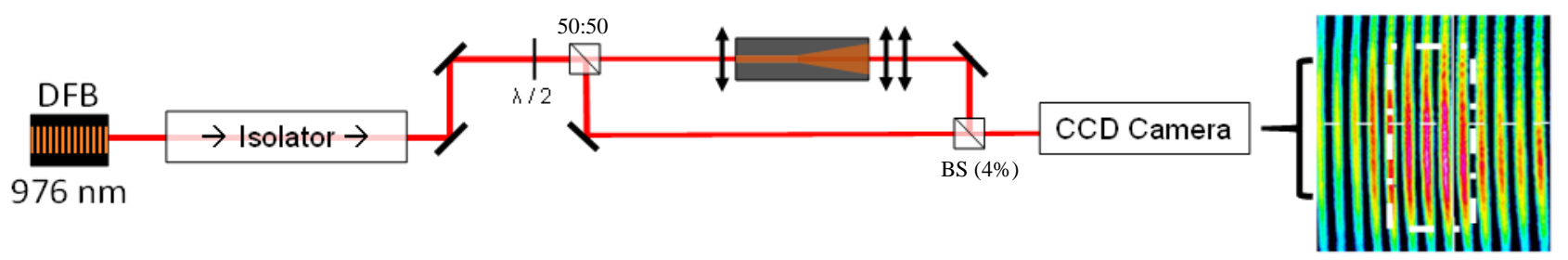

Figure 2: Experimental setup for phase noise measurement and interference fringe pattern

We measured the phase noise of one TPA under representative bias conditions using a Mach-Zehnder interferometer shown in Figure 2. The seed laser beam was optically isolated by Faraday isolators with a total isolation of $>50 \mathrm{~dB}$. The input beam was divided in two parts using a 50:50 beam splitter. The amplified beam was superimposed at a small angle to the reference beam using a beam sampler with about $4 \%$ reflection. This way one can achieve similar intensity levels for the amplified and reference beam leading to highly contrasted interference fringes. The image captured with a NIR-CCD camera shows a high visibility $(\mathrm{V} \approx 97 \%)$ of the fringes indicating a high degree of coherence. The position of the fringes, given by

$$
I(x, y)=\frac{1}{2} \times\left[I_{1}+I_{2}+2 \sqrt{I_{1} I_{2}} \cos (\Delta \Phi(x, y))\right],
$$

is directly related to the relative phase $\Delta \Phi$ between the two arms of the interferometer. Assuming that the phase in the passive reference arm is stable one can use these interference fringes to measure the induced phase fluctuations in the TPA. To do so, the camera was then replaced by two photodiodes placed in quadrature configuration, meaning with a relative shift of $\pi / 2$, in the interference fringes. The signal of the photodiode was recorded at representative bias conditions $\left(\mathrm{P}_{\text {in }}=\right.$ $10 \mathrm{~mW}, \mathrm{I}_{\mathrm{rw}}=400 \mathrm{~mA}, \mathrm{I}_{\mathrm{tp}}=7 \mathrm{~A}$ ). The measured phase fluctuations are shown in Figure 3. We note that the phase-drift over an observation time of $200 \mathrm{~s}$ is of the order of $8 \mathrm{rad}$. However, the fluctuations of the phase are slow with a standard deviation of $\sigma(\Phi)=0.1 \mathrm{rad} \approx \pi / 30$ over one second.

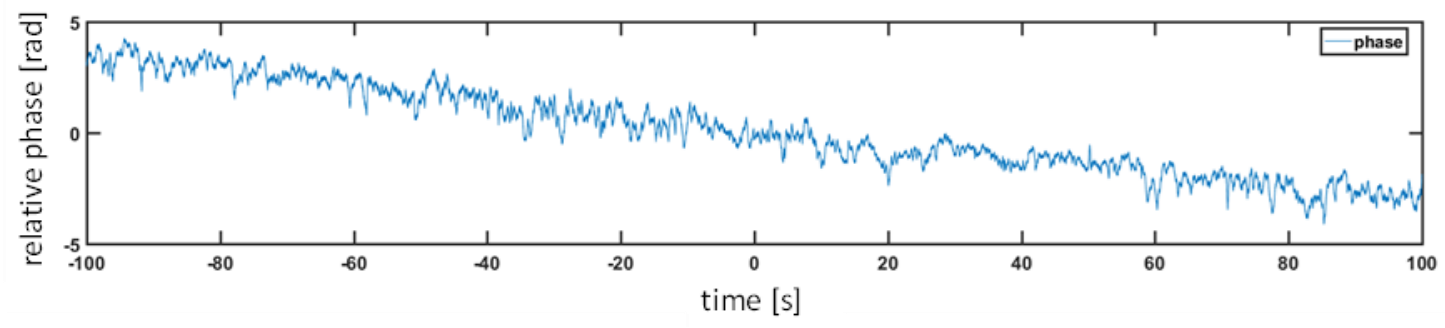

Figure 3: Measured drift of the relative phase in one TPA at $\mathrm{I}_{\mathrm{tp}}=7 \mathrm{~A}, \mathrm{I}_{\mathrm{rw}}=400 \mathrm{~mA}, \mathrm{P}$ in $=10 \mathrm{~mW}$ and $\mathrm{T}=20^{\circ} \mathrm{C}$

The corresponding phase noise spectral density $S_{\varphi}(f)$ is shown in Figure 4 (left) and confirms the domination of low frequencies ( $\mathrm{f}<10 \mathrm{~Hz}$ ) indicating that the observed phase noise is probably linked to thermal fluctuations in the TPA. The recorded phase noise was far above the corresponding background noise level of the measurement setup which was below $1 \mathrm{kHz}$. The low frequency character of the phase noise in semiconductor laser amplifiers is one advantage with respect to the phase noise of fiber amplifiers, where high frequency phase control is required in CBC-setups ${ }^{3}$. The integrated phase noise $S_{\text {int }}(F)$ 


$$
S_{\text {int }}(F)=\int_{f}^{+\infty} S_{\varphi}(f) d f,
$$

is shown in Figure 4 (right) and increases strongly with the current in the tapered section. The level of $\pi / 30$ is reached at about $1 \mathrm{~Hz}$ for at a drive current of $7 \mathrm{~A}$.
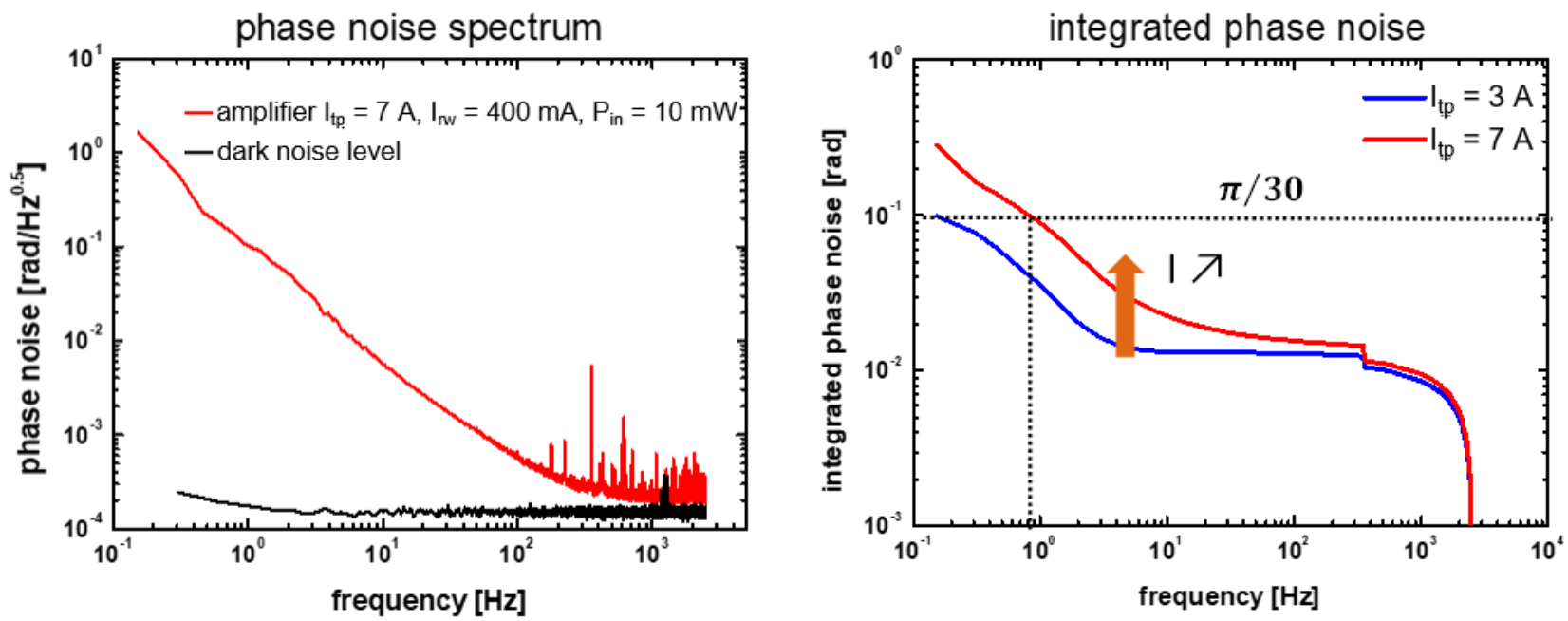

Figure 4: Phase noise spectrum of one TPA at $\mathrm{I}_{\mathrm{tp}}=7 \mathrm{~A}, \mathrm{I}_{\mathrm{rw}}=400 \mathrm{~mA}, \mathrm{P}_{\mathrm{in}}=10 \mathrm{~mW}$ and $\mathrm{T}=20^{\circ} \mathrm{C}$ and dark noise level (left) and corresponding integrated phase noise compared to a reference measurement at lower drive current $\left(\mathrm{I}_{\mathrm{tp}}=3 \mathrm{~A}\right)$ (right)

As presented earlier in section 2.1, one can use active adjustment of the ridge current in the range of 250 to $400 \mathrm{~mA}$ to control the phase pistons of the amplifier with limited impact on the output power. Regarding the requirements for the active phase control we can conclude that a simple control of the phase pistons by adjusting the ridge currents is sufficient to ensure stable $\mathrm{CBC}$ with less than $\pi / 30$ phase mismatch in the amplifiers.

\section{EXPERIMENTAL SETUP}

We recently reported on MOPA-CBC using a mini-array of comparable devices ${ }^{12}$. While experimental setups using arrays of amplifiers are an elegant solution to reduce phase fluctuations between the individual elements on the array, the experimental setup requires large diameter optics and non-standard diffractive optical elements (DOE) for splitting and recombining the beams. Lenses with a relatively long focal length $(\mathrm{f} \approx 100 \mathrm{~mm})$ are usually required while working with DOE in such experimental setups. The potential of an integration in small-footprint modules is much higher for individually mounted elements and separation and combination of the beam with standard beam splitters.

We present MOPA-CBC of three individual amplifiers in a simple experimental setup shown in figure 5, based on a multiarm Mach-Zehnder interferometer. The MO is a narrowband distributed feedback (DFB) diode laser $(\lambda=976 \mathrm{~nm})$ that is optically isolated by Faraday isolators with a total isolation of $>50 \mathrm{~dB}$. The beam is split and recombined by standard 50:50 beam splitters in three arms. The setup is insensitive to the resulting imbalances of the input power if the input power in each arm is above $10 \mathrm{~mW}$ (Figure 1). The beam was coupled in the RW by aspheric lenses with a focal length of $8 \mathrm{~mm}$. The width of the RW was $4.5 \mu \mathrm{m}$ for amplifier $\mathrm{A}_{1}$ in the upper arm and $5 \mu \mathrm{m}$ for the two other amplifiers $\mathrm{A}_{2}$ and $\mathrm{A}_{3}$, limited by the availability of the devices. Each amplifier was mounted identically as described in section 2.1. The highly astigmatic amplified beam was collimated in fast axis (FA) with an aspheric lens with a focal length of $2.75 \mathrm{~mm}$ and in slow axis (SA) with an acylindrical lens with a focal length of $18 \mathrm{~mm}$ leading to slightly elliptical beams at the output. The SA-collimation was corrected manually when the current $\mathrm{I}_{\mathrm{t}}$ in the amplifiers was changed in order to ensure a good overlap of the beams on the combining elements at each current. The beams $A_{1}$ and $A_{2}$ were combined on a first standard 50:50 beam splitter and the combined beam was then overlapped with the third $\operatorname{arm} \mathrm{A}_{3}$ on a second 50:50 beam splitter. Regarding the asymmetry of the setup, we note that the 50:50 coating of the second beam splitter is not optimal for this setup but the 
expected impact on the achievable combining efficiency is below 3\%. The optical power was measured simultaneously at the output (combined beam, $\mathrm{P}_{\text {out }}$ ) and the two unusable ports (combining losses, $\mathrm{L}_{1}$ and $\mathrm{L}_{2}$ ) and the combining efficiency was defined as $\eta=P_{\text {out }} /\left(P_{\text {out }}+L_{1}+L_{2}\right)$. The heatsink temperature was controlled individually for each amplifier. The accumulated phases $\varphi_{1-3}(t)$ in each arm of the amplifier therefore do not have a constant relation. We used the first arm $\mathrm{A}_{1}$ of the amplifier in free-running configuration and used a closed loop for phase stabilization in the other arms by changing the currents $\mathrm{I}_{\mathrm{rw}}$ of $\mathrm{A}_{2}$ and $\mathrm{A}_{3}$. A small part $(<1 \%)$ of the output beam was sent to a fast photodiode and the control loop was implemented in a microcontroller and using a sequential hill-climbing algorithm with adaptive steps described in the following section.

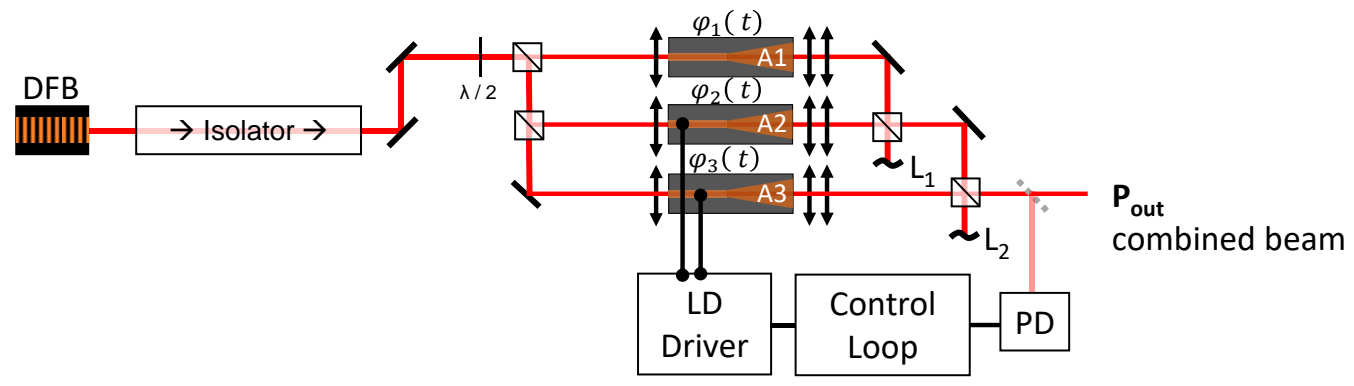

Figure 5: Experimental setup of MOPA-CBC in a multi-arm interferometer

\section{EXPERIMENTAL RESULTS \& ANALYSIS}

\subsection{Phase control and stability}

The expected phase fluctuations in the amplifiers are at very low frequencies (1-100 Hz) as shown in Figure 4. It is therefore sufficient in our case to use a sequential hill-climbing algorithm, where the relative phases $\Delta \varphi_{2}(t)=\varphi_{1}(t)-\varphi_{2}(t)$ and $\Delta \varphi_{3}$ $(t)=\varphi_{1}(t)-\varphi_{3}(t)$ are minimized sequentially. In order to do so, each optimization step in one arm of the amplifier has to be fast enough so that the phase in the other arm of the amplifier can be considered as constant. The algorithm is implemented on a fast microcontroller and uses a small perturbation of the current in both directions (dither) to calculate the local slope. The next hill climbing step is then calculated as a function of the observed slope similar to the step size in the more complex stochastic parallel gradient descent algorithms used for CBC of large arrays of devices ${ }^{3}$. If the observed slope is lower than a defined value (linked to the noise level of the measurement setup), this is taken to mean that the local maximum is reached. The same procedure then continues with the next amplifier. The dither amplitude is variable and is reduced when the system has converged in order to reduce the residual intensity fluctuations in the combined beam. Furthermore, after a certain time constant the dither amplitude is increased for a convergence test in order to avoid the system being trapped in a local minimum. The bandwidth of the feedback-control loop used in our experiments is limited by the bandwidth of the laser diode driver to about $1 \mathrm{kHz}$, which is more than sufficient to correct for the residual phase fluctuation in our experimental setup, for all drive currents used.

Figure 6 shows an example for the stabilization of the CBC power using the described algorithm for three amplifiers at a current of $\mathrm{I}_{\mathrm{tp}}=7 \mathrm{~A}$. The setup was in free-running configuration at the beginning of the measurement leading to high fluctuations of the medium output power range. The control loop was then closed after about 3 minutes and one can see that the combined power very quickly reaches its maximum power level of about $9 \mathrm{~W}$ (rise time $<1 \mathrm{~s}$ ). This power level is maintained with good stability for about one hour. The standard deviation in the stabilized level was below $0.6 \%$. The controlled RW currents were recorded and show that the phase of the whole setup is quite stable with $\Delta \varphi<\pi$ over 30 min. The average value of the applied currents evolves very slowly, however the amplitude of the recorded signal shows that continuous phase control is required to correct for small fluctuations. We occasionally observed some spontaneous large scale variation in the ridge currents corresponding approximately to a $\pi$-phase shift as clearly visible for the ridge current of $\mathrm{A}_{2}$ at $\mathrm{t}=20 \mathrm{~min}$, which are not fully understood. However, overall the active phase control is capable of controlling the output power with more than sufficient stability. 


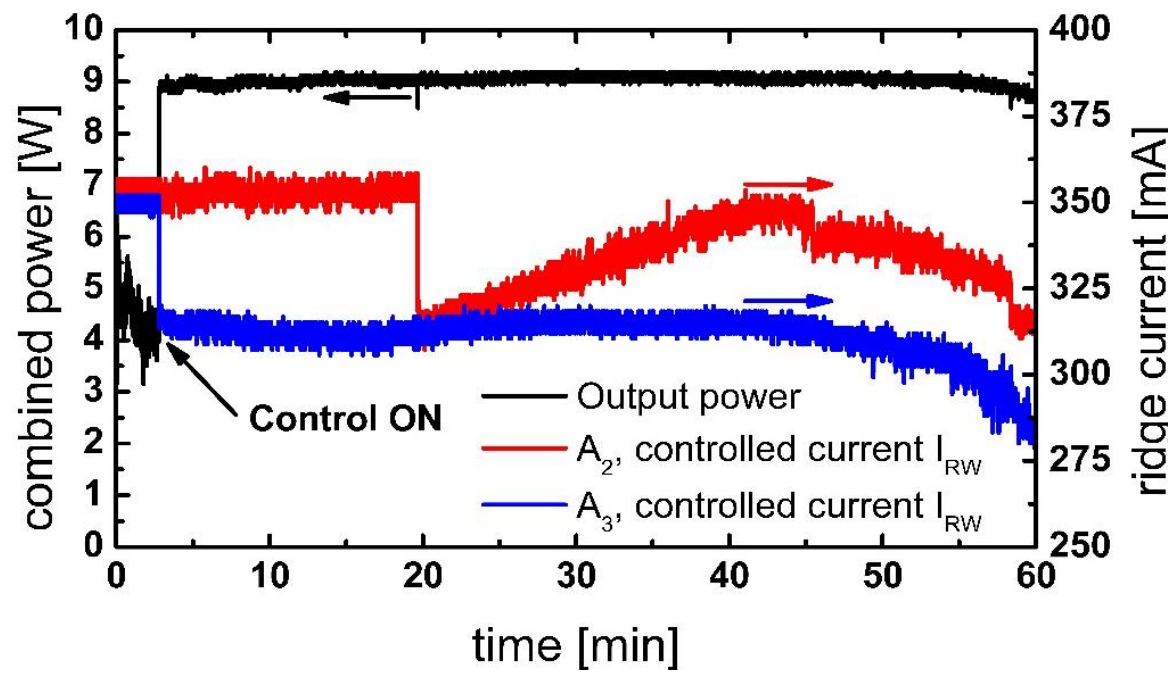

Figure 6: Combined power at $\mathrm{I}_{\mathrm{tp}}=7 \mathrm{~A}, \mathrm{~T}=20^{\circ} \mathrm{C}$ and actively regulated ridge currents of $\mathrm{A}_{2}$ and $\mathrm{A}_{3}$.

\subsection{Beam quality and combining efficiency}

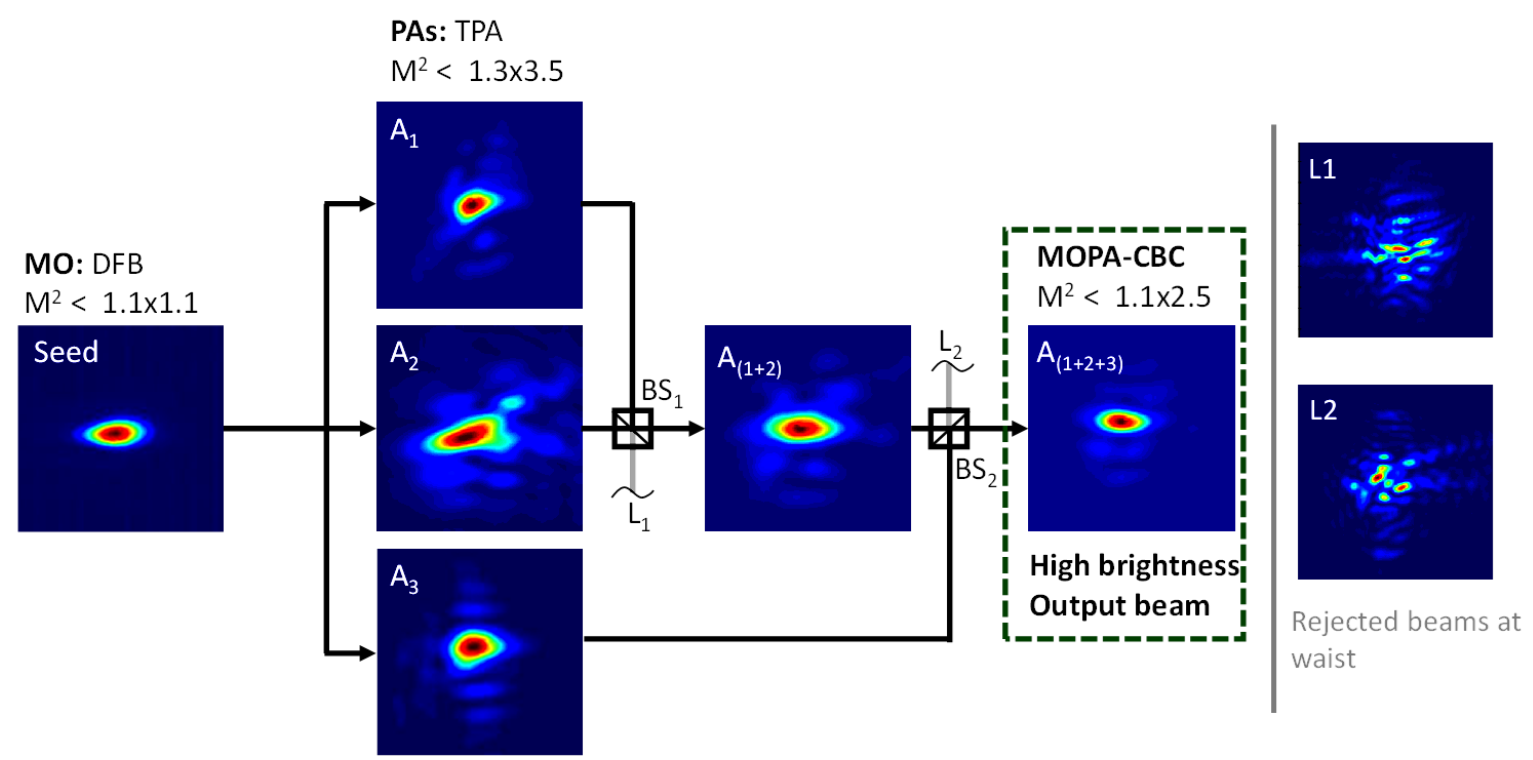

Figure 7: Beam shapes of injection beam (Seed), amplified beams $\left(\mathrm{A}_{1-3}\right)$, combined beam after first combination $\left(\mathrm{A}_{(1+2)}\right)$, combined beam at output $\left(\mathrm{A}_{(1+2+3)}\right)$ and rejected beams $\left(\mathrm{L}_{1,2}\right)$ at waist measured for $\mathrm{I}_{\mathrm{tp}}=7 \mathrm{~A}$. The FA is oriented in horizontal and the $\mathrm{SA}$ in vertical direction. Intensity is normalized in each image and shown as a linearly-scaled false color plot.

We investigated the beam quality at important positions (injection in amplifier, individual amplified beam, combined beams and rejected beams) in the experimental setup shown earlier in Figure 5. We determined the $\mathrm{M}^{2}$-parameter by fitting to measurements of development of the $4 \sigma$ beam width through the beam waist (caustic measurement). The measured shapes of the beams at the waist are summarized in Figure 7. Note that each image was normalized separately and that the exposure times were adapted depending on the reached intensity levels. In other words, the false-color scale is not comparable from one beam shape to the others. The beam shape shown of the seed laser was measured after the aspheric focalization lens used to couple the beam into the RW of the TPA. The beam quality of the amplified beams was significantly degraded in the slow axis $\mathrm{SA}\left(\mathrm{M}^{2}<3.5\right)$ compared to the diffraction-limited beam quality of the seed laser. Furthermore, the beam quality is quite sensitive to the coupling of the seed laser, which explains the slight differences in 
the observed spatial profiles of the individual beams and the slightly decreased beam quality in FA. However, the individual beams still contain $>70 \%$ of the power in the central lobe at the highest operating currents. Coherent combining automatically leads to a clean-up of the spatial profile since the beam mismatch between slightly multimode and slightly different beams is low in the central lobe but high in the rest of the beam. The combined beam $\mathrm{A}_{(1+2)}$ after the first element of combination has a higher power content in the central lobe and the rejected beam $\mathrm{L}_{1}$ mainly contains the incoherent background with low power-content in the central lobe. After combination with beam $\mathrm{A}_{3}$ on the second beam splitter one can see that the spatial profile of the output beam $\mathrm{A}_{(1+2+3)}$ is even more cleaned up with $>80 \%$ of the power in the central lobe. The measured SA beam quality parameter was $\mathrm{M}^{2}<2.5$ with a very low background of higher order spatial frequencies. The rejected beam of the second combination shows again mostly the incoherent background and high spatial frequencies with low power content in the central lobe.
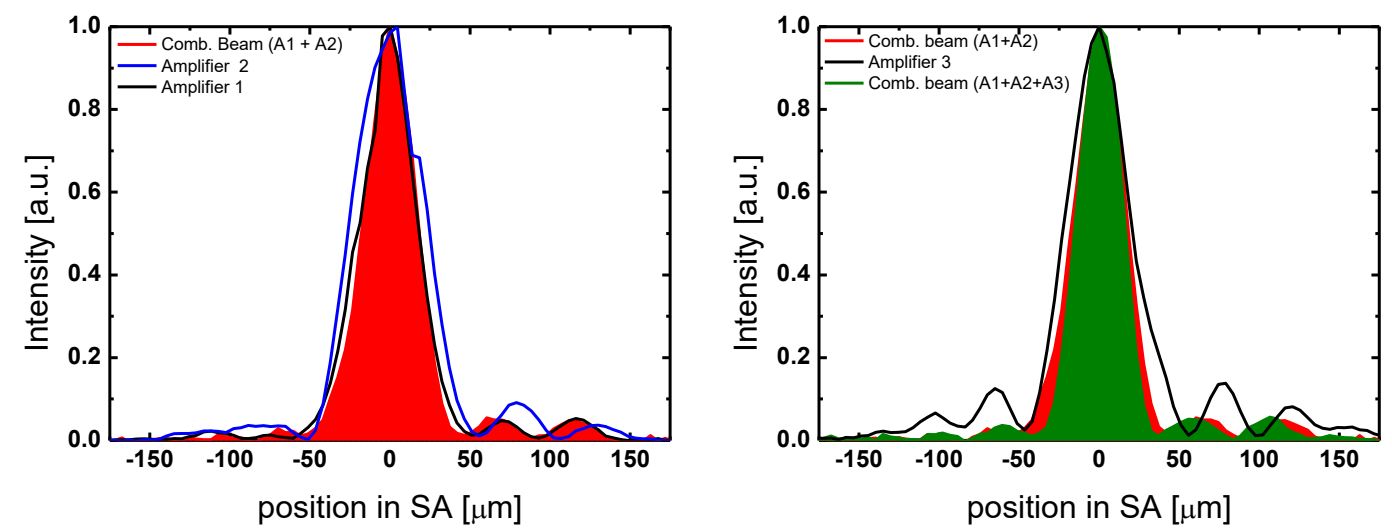

Figure 8: SA beam profiles at waist for $\mathrm{CBC}$ of $\mathrm{A}_{1}$ and $\mathrm{A}_{2}$ (left) and the following combination with $\mathrm{A}_{3}$ (right). Measurement at $\mathrm{I}_{\mathrm{tp}}=7 \mathrm{~A}, \mathrm{I}_{\mathrm{rw}}=250-400 \mathrm{~mA}$ (controlled by feedback loop).

Figure 8 shows the cross sections of the normalized intensity profile along the SA through the beam centroid of the combined beam for the combination at $\mathrm{BS}_{1}$ (left) and the following combination at $\mathrm{BS}_{2}$ (right). The profiles of the incident beams (unfilled curves for $A_{1}$ and $A_{2}$ ) profiles are slightly different but have sufficiently similar shapes for the first combination. We attribute the mismatch in the shape of the central lobes to the slightly different widths of the ridge waveguides. Nevertheless, most of the power contained in the central lobe is transferred to the combined beam (red-filled curve) while the power content in the higher order modes (power outside the central lobe) is reduced. In contrast, the incident beam profiles for the second combination are quite different. While the spatial beam profile of the beam resulting from the first combination (red-filled curve) is already cleaned-up with low power content in the higher order modes, the beam of the third amplifier has a significant power content in the side lobes. In other words, the beam mismatch seems to be significantly higher for the second combination. Almost all of the power contained in the side lobes of the third beam leads to combining losses. Additionally, the measured beam profiles indicate that not all of the power contained in the central lobe of the beam $\mathrm{A}_{(1+2)}$ is transferred to the final combined beam (green-filled curve in Figure 8(right)). Put differently, some of the power that was coherently combined on $\mathrm{BS}_{1}$ is lost during the second combining step. Furthermore, one has to take into account that the local intensities of the beam $\mathrm{A}_{3}$ and $\mathrm{A}_{(1+2)}$ are significantly different at $\mathrm{BS}_{2}$ leading to some additional combining losses in the range of $3 \%{ }^{11}$.

However, in spite of these challenges, the profile of the final output beam has $>80 \%$ power content in the central lobe. The overall combining efficiency was $70 \%$ at this operating point $\left(I_{t p}=7 \mathrm{~A}\right)$ and decreased to $65 \%$ at higher currents due to the beam quality degradation in the individual elements. For reference, the combining efficiency of the first combination, (obtained comparing the power in beam $\mathrm{A}_{(12)}$ and the losses at $\mathrm{L}_{1}$ ) was $>80 \%$ (at $7 \mathrm{~A}$ ) and is comparable to the combining efficiency which we previously demonstrated in the CBC of two individual tapered lasers in an extended-cavity configuration ${ }^{12}$. The combination on the following beam splitter $\mathrm{BS}_{2}$ leads to additional losses as explained earlier which explains the slightly reduced overall combining efficiency. We are currently seeking to improve the combining efficiency of MOPA-CBC architectures by changing the asymmetric cascade-configuration for the combination of three amplifiers described in this paper to a purely symmetric setup of an even number of individual amplifiers. In this case we expect a higher combining efficiency, since the beam mismatch in amplitude and phase will be smaller in a symmetric setup. 
Additionally, we intend to improve the achievable combining efficiency in our experimental setup by improved semiconductor design ${ }^{13}$.

\subsection{Scaling of power and brightness}

The combined power and the combining efficiency were measured for several operating points. The astigmatism of the individual amplifiers was manually corrected by moving the acylindrical lens for SA collimation with a linear translation stage. The results are summarized in Figure 9. With $12.9 \mathrm{~W}$ coherently combined power into a nearly diffraction-limited $\left(\mathrm{M}^{2}<1.1 \times 2.5\right)$ beam at the maximum current, we are able to reach higher power levels than we previously demonstrated using CBC in MOPA-configuration for an array of five tapered lasers ${ }^{14}$. The combined power per element demonstrated here was $4.3 \mathrm{~W}$ at the highest operating current, which is more than previously demonstrated with similar individually mounted tapered lasers in a rear-side resonator architecture ${ }^{12}$ indicating the MO-based phase locking scheme used is more robust and allows us to operate the individual components at higher currents. The optical power was measured simultaneously at the output (combined beam, $\mathrm{P}_{\text {out }}$ ) and the two unusable paths (combining losses, $\mathrm{L}_{1}$ and $\mathrm{L}_{2}$ ). The combining efficiency was $75 \%$ for low power levels and decreased to $65 \%$ at the maximum output power. The total power in the combined beam is increased by a factor of 2 compared to the achievable power with one individual amplifier. But, one has to consider that the beam quality of the coherently combined beam is increased compared to the beam quality of the individual devices leading to an increased brightness. The brightness $\mathrm{B}=\mathrm{P} /\left(\lambda^{2} \times \mathrm{M}^{2}\right)$ highlights the ability of extracting high optical power $\mathrm{P}$ while maintaining a good beam quality. In our case the achieved brightness was scaled by a factor of 2.8 compared to the brightness of one individual device. So even if the power scaling suffered from combining losses one can see that the brightness was scaled efficiently by the increased beam quality at the output.

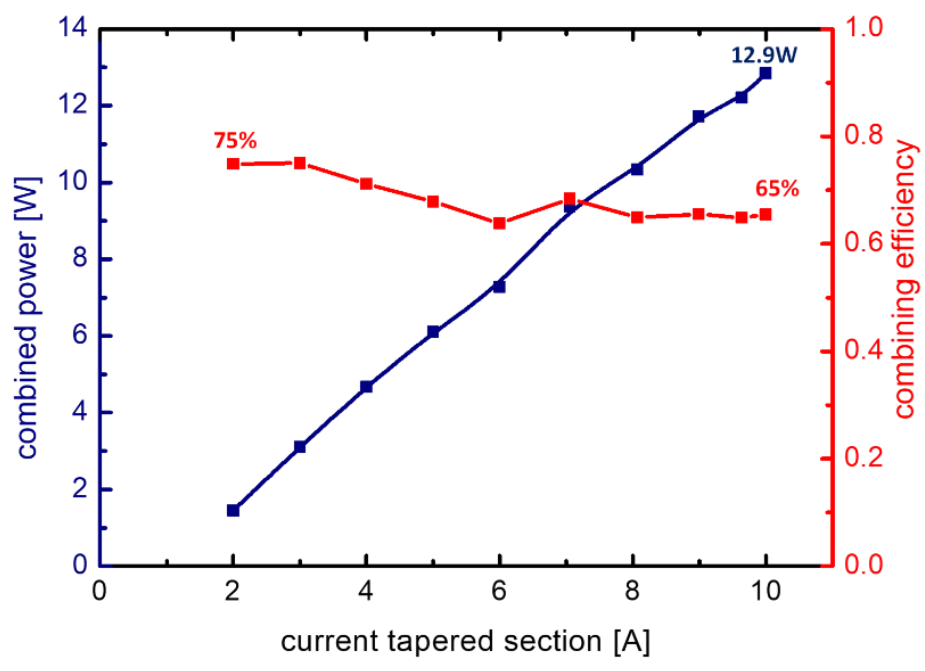

Figure 9: Combined power at output and total combining efficiency at different operating currents in the tapered section. The ridge currents were actively controlled in the range of 250 to $400 \mathrm{~mA}$.

\section{CONCLUSION}

We demonstrate the CBC of three high-power tapered laser amplifiers seeded by a DFB laser at $\lambda=976 \mathrm{~nm}$, and demonstrate a combined power of $12.9 \mathrm{~W}$ in a close to diffraction-limited beam at a combining efficiency of $>65 \%$. We used individual devices on C-mounts in a multi-arm interferometer. Increased power per element was achieved by making use of a simplified, efficiently cooled single emitter-based optical system. The combining efficiency was mostly limited by the intrinsic beam quality of the amplifiers and the asymmetric optical setup. We are currently working to improve the combining efficiency by exploiting improved semiconductor designs, better thermal management and a modified experimental setup. 
The presented results demonstrate that CBC of a low number of individual high power amplifiers is a successful and simple approach to scale the power above the limit of single emitters.

\section{REFERENCES}

[1] Fan, T., "Laser beam combining for high-power, high-radiance sources", IEEE Journal of Selected Topics in Quantum Electronics 11(3), 567-577 (2005).

[2] Witte, U., Schneider, F., Traub, M., Hoffmann, D., Drovs, S., Brand, T., Unger, A., "kW-class direct diode laser for sheet metal cutting based on DWDM of pump modules by use of ultra-steep dielectric filters", Optics Express 24(20), 22917 (2016).

[3] Brignon, A., Coherent laser beam combining, John Wiley \& Sons.

[4] McNaught, S., Thielen, P., Adams, L., Ho, J., Johnson, A., Machan, J., Rothenberg, J., Chun-Ching Shih., Shimabukuro, D. et al., "Scalable Coherent Combining of Kilowatt Fiber Amplifiers Into a 2.4-kW Beam", IEEE Journal of Selected Topics in Quantum Electronics 20(5), 174-181 (2014).

[5] Kienel, M., Müller, M., Klenke, A., Limpert, J., Tünnermann, A., "12 mJ kW-class ultrafast fiber laser system using multidimensional coherent pulse addition", Optics Letters 41(14), 3343 (2016).

[6] Creedon, K., Redmond, S., Smith, G., Missaggia, L., Connors, M., Kansky, J., Fan, T., Turner, G., Sanchez-Rubio, A., "High efficiency coherent beam combining of semiconductor optical amplifiers", Optics Letters 37(23), 5006 (2012).

[7] Albrodt, P., Hanna, M., Moron, F., Decker, J., Winterfeldt, M., Blume, G., Crump, P., Georges, P., Lucas-Leclin, G., "Coherent beam combining of high-power tapered amplifiers", 2017 IEEE High Power Diode Lasers and Systems Conference (HPD) (2017).

[8] Müller, A., Vijayakumar, D., Jensen, O., Hasler, K., Sumpf, B., Erbert, G., Andersen, P., Petersen, P., "16 W output power by high-efficient spectral beam combining of DBR-tapered diode lasers", Optics Express 19(2), 1228 (2011).

[9] Fiebig, C., Blume, G., Kaspari, C., Feise, D., Fricke, J., Matalla, M., John, W., Wenzel, H., Paschke, K. et al., "12 W high-brightness single-frequency DBR tapered diode laser", Electronics Letters 44(21), 1253 (2008).

[10] Schimmel, G., Doyen, I., Janicot, S., Hanna, M., Georges, P., Lucas-Leclin, G., Decker, J., Crump, P., Erbert, G. et al., "High-power operation of coherently coupled tapered laser diodes in an external cavity", Proc SPIE 97330I, 93480P (2016).

[11] Goodno, G., Shih, C., Rothenberg, J., "Perturbative analysis of coherent combining efficiency with mismatched lasers", Optics Express 18(24), 25403 (2010).

[12] Schimmel, G., Doyen-Moldovan, I., Janicot, S., Hanna, M., Decker, J., Crump, P., Blume, G., Erbert, G., Georges, P. et al., "Rear-side resonator architecture for the passive coherent combining of high-brightness laser diodes", Optics Letters 41(5), 950 (2016).

[13] Crump, P., Decker, J., Winterfeldt, M., Fricke, J., Maaßdorf, A., Erbert, G., Tränkle, G., "Development of high-power diode lasers with beam parameter product below $2 \mathrm{~mm} \times \mathrm{mrad}$ within the BRIDLE project", Proc. SPIE 9348, 93480D (2015).

[14] Schimmel, G., Janicot, S., Hanna, M., Decker, J., Crump, P., Erbert, G., Witte, U., Traub, M., Georges, P. et al., "Coherent beam combining architectures for high power tapered laser arrays", Proc. SPIE 10086, 1008600 (2017). 